Fischwirtschaft und Klimawandel

\title{
Herausforderungen und Chancen einer nachhaltigen Fischbewirtschaftung
}

\begin{abstract}
Der Klimawandel stellt die Fischwirtschaft vor neue Herausforderungen, denn klimawandelbedingte Veränderungen wirken als zusätzliche Stressfaktoren auf das marine Ökosystem. Für Unternehmen und Konsumenten kann der Klimawandel jedoch ganz unterschiedliche Auswirkungen hervorrufen. Von Marina Beermann
\end{abstract}

W eltweit gewinnt die Ressource Fisch als Proteinlieferant für die Ernährungsversorgung und -sicherung neben der Fleischproduktion zunehmend an Bedeutung. Die Anfang 2009 erschienene Studie der Ernährungs- und Landwirtschaftsorganisation der Vereinten Nationen (FAO) zum weltweiten Stand der Fischerei und Aquakultur hat hierbei aktuelle Studien zusammengetragen und neben dem Einflussfaktor Klimawandel, wesentliche Entwicklungen im Konsum von Fischprodukten analysiert.

Während in Entwicklungsländern, insbesondere in der Mittelklasse der Länder Ost- und Südostasiens, die Nachfrage nach eiweißreichen Fischprodukten insgesamt steigt, setzt sich in den Industrieländern der Trend zu einer weiteren Qualitätsdifferenzierung des Lebensmittels Fisch fort. Der Wunsch nach einer vielfältigen, ausgewogenen und damit gesunden Ernährung gewinnt parallel zum weiter steigenden Trend zu Fertigprodukten und der Einhaltung hoher Standards bezüglich der Lebensmittelsicherheit an Bedeutung.

\section{Fischwirtschaft im Übergang}

Die Forderung nach erhöhter Transparenz durch erweiterte Zertifizierungen im Bereich von Umwelt- und Tierschutzaspekten wird begünstigt durch steigende Löhne, steigendes Bevölkerungswachstum und weitere Urbanisierung in den Industrieländern. Darüber hinaus setzt sich die rasante Entwicklung im Bereich der Aquakultur weltweit fort, wobei diese mengenmäßig von den Ländern China und Vietnam angeführt wird (FAO 2009).

Für Akteure der Fischerei und Fisch verarbeitender Unternehmen sind diese Entwicklungen entscheidend für die weitere strategische Ausrichtung und wird einerseits bedingt durch die Anpassungsfähigkeit des Unternehmens und andererseits durch die Fähigkeit pro-aktiv Veränderungen strategisch nutzen zu können. Doch welchen Einfluss werden der Klimawandel und damit einhergehende Veränderungen sowohl auf Unternehmen der Fischwirtschaft als auch auf Konsumenten des Naturprodukts Fisch ausüben?

Die im Dezember 2008 vom Bundeskabinett beschlossene Deutsche Anpassungsstrategie an den Klimawandel (DAS) hat zum einen die Dringlichkeit aufgezeigt, Antworten auf klimawandelbedingte Veränderungen finden und entwickeln zu müssen, und zum anderen die Heterogenität der vom Klimawandel betroffenen Lebens- und Wirtschaftsbereiche verdeutlicht.

Wesentliche klimawandelbedingte Einflussfaktoren für die Fischerei und die Fisch verarbeitenden Unternehmen sind hierbei ein Anstieg der Meerestemperatur, Schwankungen der Salinität sowie eine Verschärfung von Wind- und Seegangsbedingungen für die Schifffahrt. Diese Faktoren beeinflussen neben den Bedingungen für die Durchführung des Fischfangs, auch die Voraussetzungen für ertragreiche Fangquoten. Anhand der Erläuterung potenzieller Folgen eines An- stiegs der Meerestemperatur kann exemplarisch die Tragweite möglicher Veränderungen verdeutlicht werden:

I Eine vermehrte Produktion von Biomasse erhöht durch dessen Abbau die Sauerstoffverzehrung und führt $\mathrm{zu}$ Störungen innerhalb des Nahrungskettengleichgewichts und kann in einem Rückgang von Fisch- und Muschelpopulationen resultieren.

- Ein sprunghafter Anstieg von toxischen Substanzen und Algen kann sich negativ auf die Entwicklung von Fisch- und Muschelbeständen auswirken.

- Es muss mit einem Rückgang von bestimmten Fischarten gerechnet werden, insbesondere bei Beständen, deren Jungfische temperatursensibel sind.

- Eine Veränderung des Verhältnisses zwischen Räuber und Beute.

- Ein Eindringen fremder Fischarten kann zu einer Neubesetzung von ökologischen Nischen führen.

Für die produzierenden Unternehmen der Fischwirtschaft können diese potenziellen klimawandelbedingten Veränderungen des marinen Ökosystems weitreichende Folgen auf die betrieblichen Prozesse haben. Grundsätzlich sind die fischwirtschaftlichen Unternehmen abhängig von den natürlichen regionalen und internationalen Fischvorkommen.

\section{Herausforderungen für Unternehmen}

Klimawandelbedingte Veränderungen der weltweiten Verfügbarkeiten bestimmter Fischarten und -mengen können es erforderlich machen, alternative Fischarten sowie daran angepasste Produktionsweisen und Produkte zu entwickeln, die sowohl den Ansprüchen der Anbieter- als auch der Nachfragerseite gerecht werden müssen. Dabei gilt es aus unternehmerischer Perspektive zu berücksichtigen, dass die Konsumenten einerseits zwar vermehrt Fisch als gesunde Ernährungsvariante zu Fleisch nachfragen, andererseits jedoch primär zu Fischprodukten greifen, deren Geschmack bekannt und wenig fischintensiv ist. 
Die Unternehmen der Fischwirtschaft sind damit vor diverse Herausforderungen gestellt. Sie müssen natürliche Schwankungen der Fischpopulationen sowohl in der Quantität als auch in der Qualität in ihre Unternehmens- und Produktionsplanungen einbeziehen. Unter Klimawandelgesichtspunkten ein bisher schweres Unterfangen, da die klimatischen Veränderungen sich zum einen sehr unterschiedlich auf die diversen Fischbestände auswirken können und zum anderen bisher hohe Unsicherheit bezüglich der Bestandsprognosen und der Managementpläne existieren. Darüber hinaus gilt es aus unternehmerischer Perspektive, die Unvorhersehbarkeit von Störungen innerhalb der Fischpopulation, des marinen Ökosystems insgesamt und der globalen Fischmärkte zu kalkulieren.

\section{Ökologische Prozesse beeinflussen unter- nehmerische Planungen}

Die Bedeutung und Tragweite der gegenseitigen Wechselwirkungen von natürlichen ökosystemaren Prozessen und unternehmerischen Planungen wird mit dem Fokus auf die Fischwirtschaft besonders deutlich. Vor dem Hintergrund des Klimawandels stellt sich verstärkt die Frage, inwiefern Systeme möglichst anpassungs- und widerstandsfähig gestaltet werden können, ohne essenzielle Strukturen und Funktionen des Systems zu verlieren. Diese Art Pufferkapazität eines Systems wird als Resilienz bezeichnet und gewinnt im Zuge der internationalen Klimawandeldiskussionen zunehmend an Bedeutung. Für eine langfristige Sicherung und nachhaltige Nutzung der weltweiten Fischbestände kann das Konzept der Resilienz hilfreiche Erkenntnisse liefern.

Die Resilienz von auf dem marinen Ökosystem basierenden und davon abhängigen Systemen, wie dem der Fischwirtschaft, wird unmittelbar durch die Widerstandsfähigkeit und Robustheit des Ökosystems selbst bestimmt. Bereits bestehende Stör- und Stressfaktoren, die auf das marine Ökosystem Einfluss nehmen wie beispielsweise die Überfischung, üben darüber hinaus Einfluss aus auf die Fähigkeit resilient auf zusätzliche klimawandelbedingte Veränderungen reagieren zu können. Für die Sicherung der Produktvielfalt des Lebensmittels Fisch insgesamt gilt es, bereits bestehende negative Einflussfaktoren zu reduzieren, um die Pufferkapazität für bisher noch nicht absehbare klimawandelbedingte Auswirkungen nicht zusätzlich zu belasten. Eine nachhaltige menschliche Nutzung natürlicher Ressourcen kann somit die Resilienz eines Ökosystems positiv beeinflussen und damit die Fähigkeit, Störfaktoren abpuffern zu können, erhöhen. Dies erscheint insbesondere vor dem Hintergrund einer wachsenden Weltbevölkerung unumgänglich zu sein.

\section{Zukunftsfähige Fischwirtschaft}

Zukunftsfähige Lösungsansätze für eine nachhaltige Fischbewirtschaftung sind vor dem Hintergrund der komplexen Strukturen des marinen Ökosystems einerseits und der fischwirtschaftlichen Strukturen andererseits nicht allgemeingültig definierbar. Dennoch gibt es einige Ansatzpunkte, die helfen können, die Resilienz bestimmter Prozesse im Rahmen der Fischwirtschaft zu erhöhen.

Sowohl die Fischindustrie als auch der Fischhandel müssen mehr Transparenz hinsichtlich der Produktkennzeichnung garantieren. Fehlende Informationen sowie mangelnde Fachberatung machen es verantwortungsbewussten Konsumenten schwer, die richtige Wahl beim Fischkauf zu treffen. Die im Sommer 2008 vom Verband der Fischindustrie und des Fischgroßhandels verabschiedete erweiterte Fanggebietskennzeichnung geht hierbei in die richtige Richtung.

In den Stufen des Fischfanges und der -verarbeitung gilt es, mehr Effizienz in Bezug auf die Fangmethoden und -richtlinien walten zu lassen. Das Problem des Beifanges ist dabei vor allem eine politische Frage. Die rasante Entwicklung der Aquakultur muss vor dem Hintergrund steigenden Wachstums weiter im Sinne einer nachhaltigen Nutzung optimiert werden. Hoffnungsvolle Wege für die Bio-Zertifizierung gehen dabei bereits die Vermarkter Naturland und Bioland.

Aufseiten der Konsumenten muss sich vergleichbar zum Fleischkonsum auch beim Kauf von Fischprodukten in westlichen Ländern vermehrt ein Paradigmenwechsel hin zu mehr Klasse, statt Masse vollziehen. Wertschätzung des Lebensmittels und ein bewusster Konsum, unter anderem durch mehr Transparenz und detaillierte Informationen, sind hier notwendig.

Klimawandelbedingte ökologische Diskontinuitäten werden zunehmend weitreichende Folgen für Akteure der Fischwirtschaft haben und gezielte Anpassungsmaßnahmen erforderlich machen. Eine klimawandelbedingte Differenzierung der Rohstoffbasis wird auch das Angebot für Konsumenten von Fischprodukten zunehmend in Richtung von Fisch aus Aquakulturen verschieben. Die nachhaltige Nutzung und Sicherung der Naturressource Meeresfisch gewinnt somit unter Klimawandelaspekten verstärkt an Brisanz.

I AUTORIN + KONTAKT

Marina Beermann ist Doktorandin am Lehrstuhl für Allgemeine Betriebswirtschaftslehre, Unternehmensführung und Betriebliche Umweltpolitik der Universität Oldenburg und Projektkoordinatorin des BMBF-Projektes „Nordwest 2050 - Perspektiven für klimaangepasste Innovationsprozesse in der Metropolregion Bremen-Oldenburg" des Clusters Ernährungswirtschaft.

Marina Beermann, Carl von Ossietzky Universität Oldenburg, Fakultät II, Lehrstuhl für Allgemeine Betriebswirtschaftslehre, Unternehmensführung und Betriebliche Umweltpolitik, Ammerländer Heerstraße 114-118, Gebäude A5, Raum 223, 26129 Oldenburg. Tel.: +49 4417984968 Fax: +49441794193 
(c) 20I0 Authors; licensee IÖW and oekom verlag. This is an article distributed under the terms of the Creative Commons Attribution Non-Commercial No Derivates License (http://creativecommons.org/licenses/by-nc-nd/3.o/), which permits unrestricted use, distribution, and reproduction in any medium, provided the original work is properly cited. 